\title{
Analyzing the Possibility of Utilizing CBCT Radiomics as an Independent Modality: A Phantom Study
}

\author{
Dharmendran Palani ${ }^{1 *}$, Senthilkumar Shanmugam², Kesavan Govindaraj ${ }^{1,3}$
}

\begin{abstract}
Aim: To verify if computed tomography (CT) radiomics were reproducible by cone beam CT (CBCT) radiomics by using Catphan ${ }^{\circledR} 504$. Materials and Methods: Catphan $^{\circledR} 504$ was imaged using the default IGRT OBI CBCT imaging protocols and CT scanner. Seven known density image regions of the phantom were segmented and image feature was extracted by Imaging Biomarker Explorer (IBEX) software. The 49 selected features from four feature categories were analyzed by considering each region of interest (ROI) segment as individual image set. Correlation was studies using interclass correlation coefficient (ICC) and Pearson's correlation coefficient. Results: The ICC of the three feature categories, namely intensity, GLCM, and GLRLM was significant ( $p$-value $<0.05)$ in comparison with $\mathrm{CT}$, while the ICC of the fourth feature category, NID, was no significant. The average absolute Pearson's correlation coefficient from the features of the images was as follows: $\mathrm{CT}$ : $\mathrm{r}=0.679 \pm 0.257, \mathrm{CBC}_{\text {Thead }}: \mathrm{r}=0.707 \pm 0.231, \mathrm{CBCT}_{\text {thorax }}$ : $\mathrm{r}=0.643 \pm 0.260$, and $\mathrm{CBCT}_{\text {pelvis }}: \mathrm{r}=0.594 \pm 0.276$. Conclusion: It seems that the various densities of Catphan ${ }^{\circledR} 504$ ROI image segments of the $\mathrm{CT}$ radiomics are reproducible with $\mathrm{CBCT}$ radiomics and $\mathrm{CBCT}$ radiomics can be used as an independent modality.
\end{abstract}

Keywords: Texture- quantitative imaging features- cone-beam CT- computed tomography

Asian Pac J Cancer Prev, 22 (5), 1383-1391

\section{Introduction}

The field of medical imaging provides comprehensive imaging tools. The applications of multimodality images in radiotherapy development from the recent past to the current diagnostic images were appreciated in precise delineation of target volume (TV), organ at risk (OAR), treatment plan, treatment response assessment and treatment follow up. Amongst the various multimodality images, computed tomography (CT) images plays two vital roles, namely defining pretreatment tumor and tumor responses to treatment, of which the later impacts greatly the treatment decision. However, the recent modern advancements in imaging analysis, namely radiomics, extracts additional quantitative features from medical images, such as CT, positron emission tomography (PET), and magnetic resonance imaging (MRI), to uncover patient's response to treatment as well as the chance of developing side effects (Gillies et al., 2016). $\mathrm{CT}$ radiomics is a conventionally practiced prognostic feature in radiation therapy. Various clinical, phantom, and texture analysis of CT radiomics have discussed both its reliability in assessing tumor response to treatment and its limitations. However, such limitations outweigh the established purpose of CT radiomics (Nardone et al.,
2016; Nie et al., 2016; De Cecco et al., 2016; Bundschuh et al., 2014; Pyka et al., 2015; Tian et al., 2015; Yip et al., 2014). Three - dimensional (3D) cone-beam CT (CBCT) is taken regularly for patient setup and position verification (Lambin et al., 2017). These images are then used to study the tumor changes during the course of treatment (Brink et al., 2014). Radiomics based on CBCT imaging offers a potential for primary treatment process where the prognostic value of conventional CT images is already known (Fried et al., 2014; Coroller et al., 2015; Aerts et al., 2014). There are very few literatures explaining the possibilities for image features extracted from CBCT to be a potential alternative for CT. Moreover, the prospective of radiomics in CBCT needs to be investigated particularly in the image quality of CBCT as this dimension is unfamiliar when compared to the conventional CT. Therefore, this study aimed to assess the quality of CBCT-based radiomic features with selected known image density region of interest (ROIs) and compared to it CT-based radiomics features. The parameter of interest was to analyze the gray level value changes of the ROIs image density between the $\mathrm{CBCT}$ and $\mathrm{CT}$ by computing the first order intensity and texture matrices. To assess the reliability of CBCT radiomic features, Catphan ${ }^{\circledR} 504$ phantom images of head, thorax, and pelvis image guided radiation therapy

${ }^{1}$ Research and Development Centre, Bharathiar University, Coimbatore, India. ${ }^{2}$ Department of Radiotherapy Government Rajaji Hospital \& Madurai Medical College, Madurai, Tamil Nadu, India. ${ }^{3}$ Department of Radiotherapy, Vadamalayan Hospitals Integrated Cancer Centre, Madurai, India. *For Correspondence: pdharmendran75@gmail.com 
(IGRT) protocols of on-board imaging (OBI) CBCT were obtained since phantoms have proved to provide robustness in non-invasive studies .This study further investigated the possibility of utilizing $\mathrm{CBCT}$ radiomics as an independent modality to assess the tissue equivalent density region.

\section{Materials and Methods}

\section{Catphan $^{\circledR} 504$}

Catphan ${ }^{\circledR} 504$ phantom is the most common available phantom in IGRT. It is supplied by Varian Medical Systems. This phantom is designed to monitor sensitometry target values. Over the time, it is proved to provide valuable information like indicating changes in scanner performance. It is regularly used to define the CT density table in the treatment planning system for patient dose calculation and as OBI CBCT image calibration . Catphan ${ }^{\circledR} 504$ phantom module has sensitomerty region which has known density material inserts made up of Teflon, Delrin, acrylic, polystyrene, low density polyethylene (LDPE), polymethylpentene (PMP), and air. The relative electron densities are $1.868,1.363$, $1.147,0.998,0.945,0.853$, and 0.001 , respectively. Each density insert is $1.25 \mathrm{~cm}$ diameter and $2.5 \mathrm{~cm}$ in length. This phantom was chosen for this study for its predefined known seven ROI density and easy accessibility in most of the IGRT setup as it is routinely used as a tool for determining image calibration and quality.

\section{Phantom imaging}

To determine the similarity between the CBCT radiomics texture values and those of $\mathrm{CT}$ images, we imaged Catphan ${ }^{\circledR} 504$ using the default IGRT head ,thoracic and pelvis CBCT Varian Linac OBI imaging protocols and GE medical system (Discovery IQ) CT scanner. Each CBCT scan is classified as CBCT head scan (CBCThead), CBCT thorax scan (CBCT thorax), and CBCT pelvis scan (CBCT pelvis). The characteristics of the scan parameters are given in Table 1.

\section{ROI segmentation}

The scanned images in Digital Imaging and Communication in Medicine (DICOM) format were imported into Imaging Biomarker Explorer (IBEX) software (available for download at http://bit.ly/ IBEX MDAnderson). ROI density was delineated in all the four scanned image sets using the IBEX graphical user interfaces (GUI) countering option. Delineated segmented ROI shown in Figure1 were considered to be sub images for the sources of texture feature extraction. Each ROI was manually segmented and added to the data sets which contained basic image information, such as ROI statistics, voxel, ROI contours, ROI binary masks, and image data in the ROI bounding box. As there were possibilities of inaccurate contour values, accurate countering was necessary and features were only calculated within these areas. Radiomics features extracted from the segmented density ROI volume within phantom is highlighted in Figure 2.
Table 1. Characteristics of the Scan Parameters of the CT and CBCT Images

\begin{tabular}{lcccc}
\hline Manufacturer & $\begin{array}{c}\text { GE medical } \\
\text { system CT }\end{array}$ & Varian medical system OBI \\
\hline Scan protocol & CT-Pelvis & $\begin{array}{c}\text { CBCT- } \\
\text { Head }\end{array}$ & $\begin{array}{c}\text { CBCT } \\
\text { Thorax }\end{array}$ & $\begin{array}{c}\text { CBCT } \\
\text { Pelvis }\end{array}$ \\
Image size (pixels) & $512 \times 512$ & $384 \times 384$ & $384 \times 384$ & $384 \times 384$ \\
Pixels size (mm) & 0.0976 & 0.0625 & 0.1171 & 0.1171 \\
$\begin{array}{l}\text { Slice thickness } \\
\text { (mm) }\end{array}$ & 2.5 & 2.5 & 2.5 & 2.5 \\
Tube Voltage (kVp) & 120 & 100 & 110 & 125 \\
$\begin{array}{l}\text { Exposure time } \\
\text { (mAs) }\end{array}$ & 6 & 145 & 262 & 680 \\
Tube current (mA) & 300 & 20 & 20 & 80 \\
Scanner rotation & Helical & Half fan & Full fan & Full \\
& & $200^{\circ}$ & $360^{\circ}$ & fan360 \\
Filter & & Full & Half & Half \\
& & bowtie & bowtie & bowtie \\
& & filter & filter & filter \\
No of image Projection & & 360 & 655 & 655 \\
\hline
\end{tabular}

\section{Radiomics feature definition and extraction}

ROI data of four image sets were preprocessed with histogram equalization enhancement preprocessing algorithm. The four-feature categories and 49 features extraction algorithm were developed in the feature algorithm workspace in IBEX. The selected 49 features can be generated for different applications, such as tumor diagnosis, tumor staging, gene prediction, and outcome prediction.

The selected feature categories for this phantom study were 11 Intensity Histogram and Gray Level Run Length Matrix, 22 Gray level Co-occurrence Matrix, and 5 Neighborhood Intensity Difference matrix, as shown in the Table 2.

\section{Statistical analysis}

The feature category clusters, namely intensity,

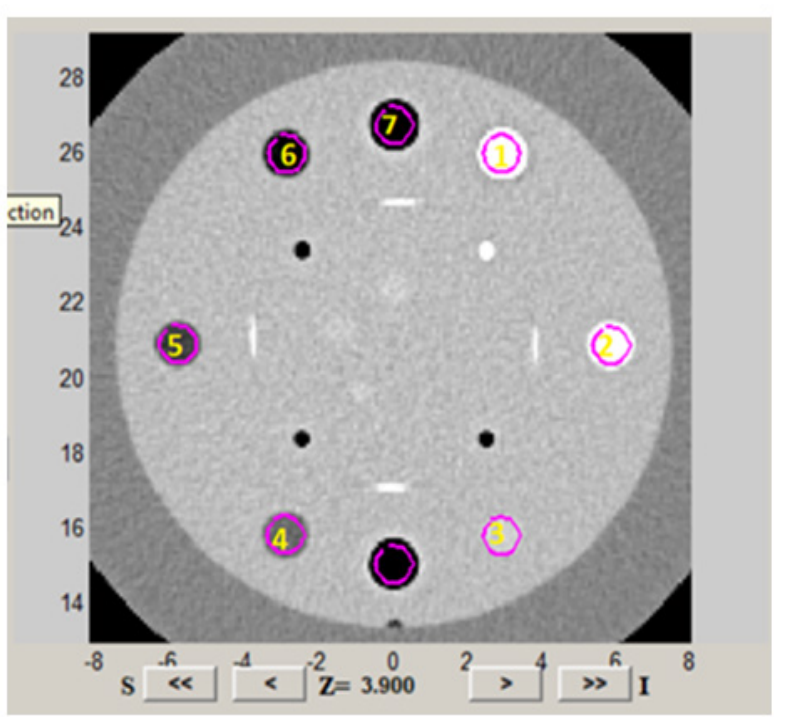

Figure 1. Axial Section Taken for the Analysis of CT Catphan ${ }^{\circledR} 504$ Sensitometry Region Image with Segmented ROI Known Density Materials 1)Teflon , 2) Delrin , 3) Acrylic ,4) Polystyrene , 5) LDPE, 6) PMP and 7) Air 


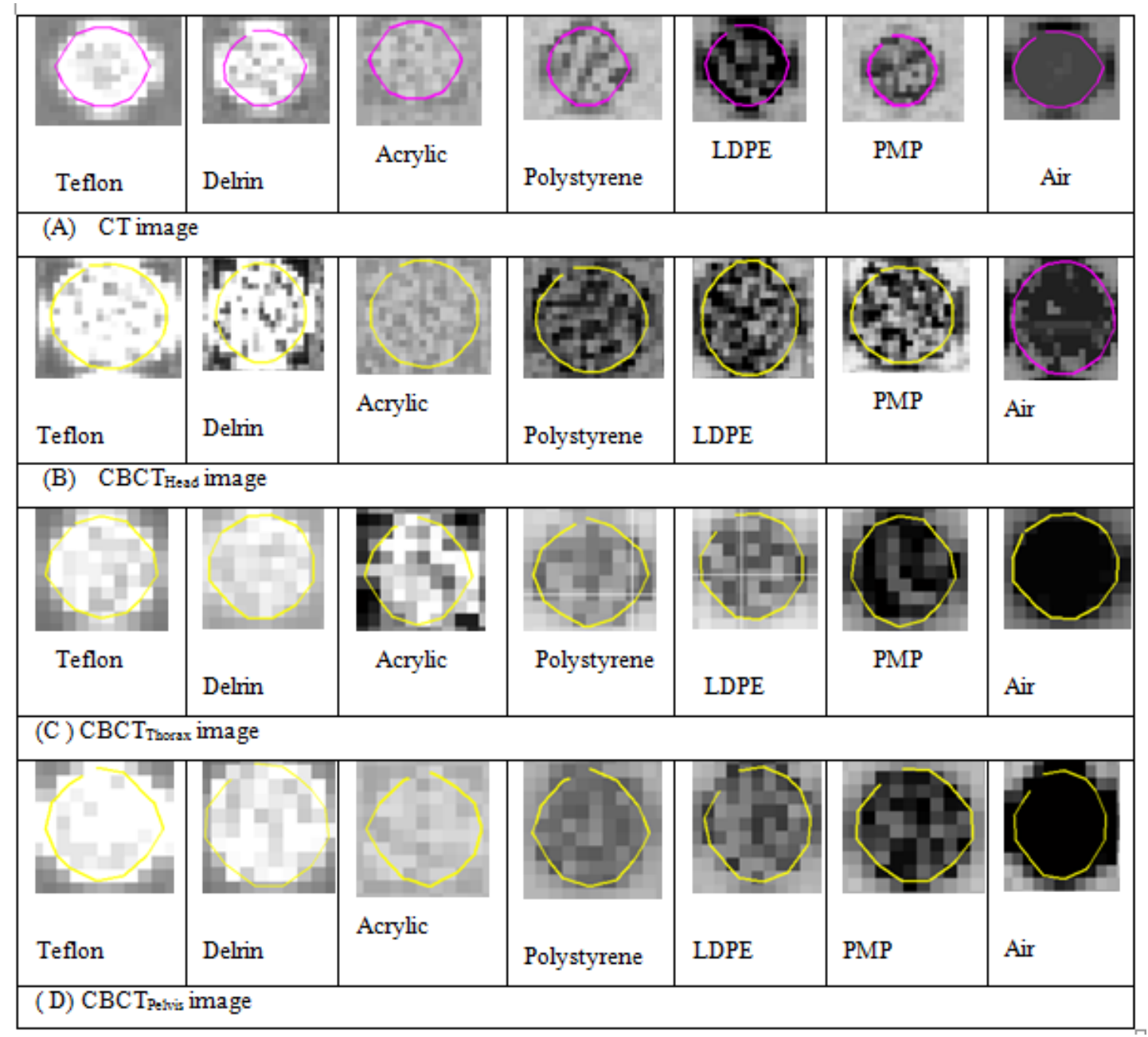

Figure 2. Different ROI Density Sensitomerty Regions Catphan ${ }^{\circledR} 504$ (A) CT Image (B)-(D) CBCT Images

GLCM, GLRLM, and NID radiomics feature values, extracted from individual density ROIs image data set were selected for the analysis. The interrelation relatability between the images was calculated using intra-class correlation coefficient (ICC). Variance estimates were obtained using two-way mixed effects and absolute agreement method. The ICC is a statistical measure ranging from 0 to 1 , indicating null and perfect reproducibility, respectively. The ICC $>0.7$ is considered significant in the interrelated reproducibility between the CT and CBCTs. The bivarFiate Pearson's correlation coefficient was used to analysis the density dependent related changes of the extracted radiomics features from the known seven ROIs present within the $\mathrm{CT}$ and CBCT. The correlation coefficient value ranges from \pm 1 to 0 in which -1 and +1 indicates perfect negative and perfect positive, respectively. In this condition, $r=0$ (zero relationship) implies no correlation, $0.1<|\mathrm{r}|< \pm 0.3$ is said to show small / weak correlation, while the values between $\pm 0.3<|\mathrm{r}|< \pm 0.5$ medium / moderate correlation and $\pm 0.5<|\mathrm{r}|$ is large / strong correlation. In this study, the ICC and Pearson's correlation coefficient were analyzed using the IBM SPSS.

\section{Results}

The radiomics features extracted using IBEX from seven known phantom density ROIs image data set were concurrently analyzed with $\mathrm{CBCT}$ inter scan IGRT imaging protocol ROIs image segment radiomics feature category cluster reproducibility, and radiomics feature density dependency consistency was compared with known CT features.

\section{CBCT interrelation relatability with CT image}

Radiomics feature category clusters, namely intensity, GLCM, GLRLM, and NID, were extracted from the seven density ROIs segments. Each ROI was considered as individual image feature data set. The ICC was calculated for the ROI feature category clusters of CBCT and CT images, and the results are expressed in Table 3. The average ICC of the feature category cluster was significant ( $\mathrm{ICC}=0.913 \pm 0.123, \mathrm{P}=0.011$ ). Correlation value more than $>0.7$ is considered as strong interrelated repeatability among the images. ICC analysis result of the first three radiomics cluster features indicated significant $\mathrm{CBCT}$ inter scan IGRT imaging protocol interrelated repeatability with CT. However, NID cluster feature value from acrylic and air ROIs had ICC $=0.418(\mathrm{P}=0.209)$ and $\mathrm{ICC}=0.607$ $(\mathrm{P}=0.09)$, respectively, revealing less correlation significance. Each ROI segment cluster feature category 


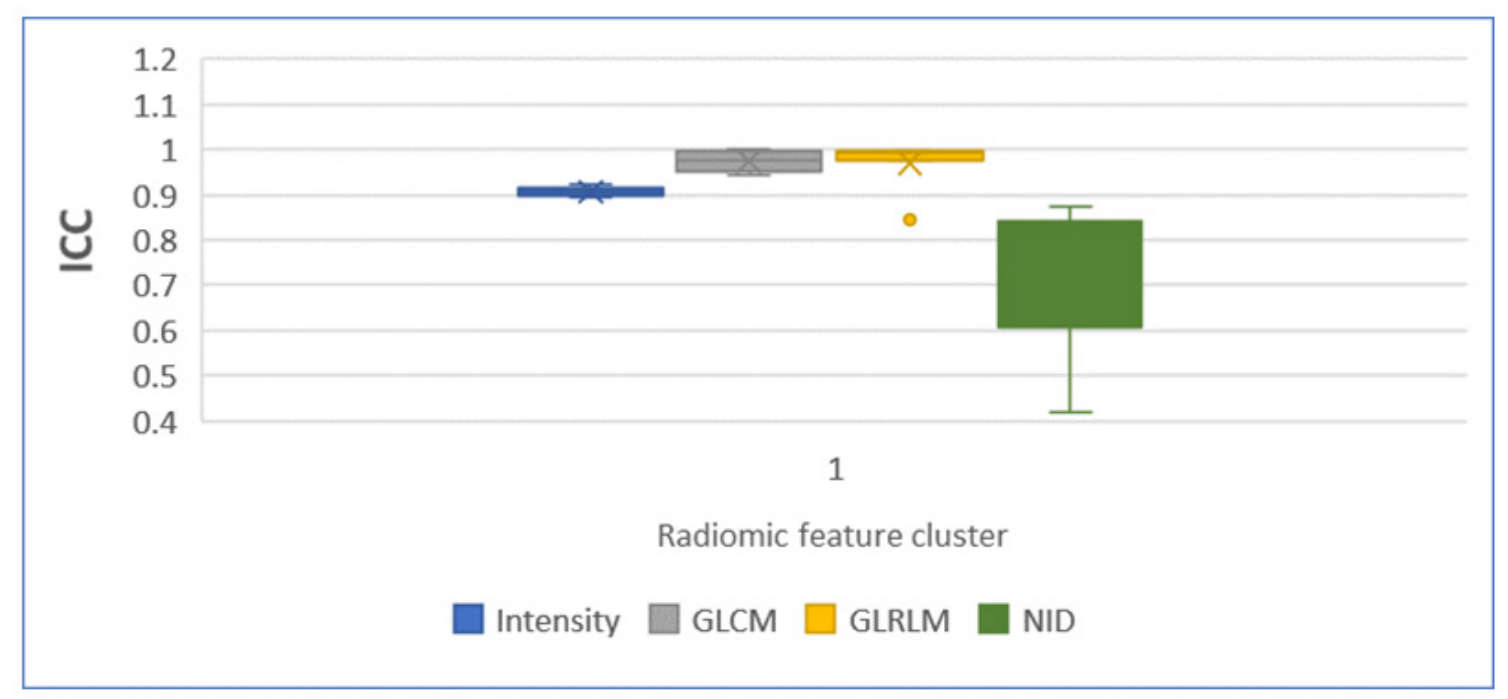

Figure 3. Box Plot Wispier of Radiomics Feature Cluster vs. ICC

ICC comparison is shown in Figure 3.

\section{ROI density radiomics feature correlation consistency} among the images

The 49 computed features correlation strength significance and magnitude were compared among the images. Here, the Pearson's scoring of the four image sets, namely CT, CBCThead, CBCTthorax , and CBCTpelvis, was considered to be the similarity predictors. The average absolute Pearson's correlation coefficient from the features of the images was as follows: CT: $r=0.679 \pm 0.257$ ( 40 of the 49 features had $r>0.5$ and $55 \%$ of the selected features had significant ( $p$-value $<0.05)$ ), $\mathrm{CBCT}_{\text {head }}: \mathrm{r}=0.707 \pm 0.231$ (39 of the 49 features had $\mathrm{r}>0.5$ along with $59 \%$ of the selected showed the significance (p-value $<0.05)$ ), $\mathrm{CBCT}_{\text {thorax }}: \mathrm{r}=0.643 \pm 0.260$ (35 of the 49 features had $r>0.5$ with $43 \%$ significance $(p$-value $<0.05)$ ), and $\mathrm{CBCT}_{\text {pelvis }}: \mathrm{r}=0.594 \pm 0.276$ (27 of the 49 features had $\mathrm{r}>0.5$ and $41 \%$ significance ( $\mathrm{p}$-value $<0.05)$ ). Nineteen computed features out of the 49 selected radiomics features from all the four image sets had very strong correlation $(r>0.750)$ and were significant $(p$-value $<0.05)$ as shown in the Figure 4(a-d). The results of Pearson's correlation coefficient test on the image radiomics features are presented in Table 4 (a-d).

\section{Discussions}

In this phantom study, amongst the four inter scan image modalities, CBCThead radiomics features, 80\% of the 49 selected features demonstrated strong correlation $\mathrm{r}>0.5$, and $55 \%$ of the features were significant ( $\mathrm{p}$-value $<0.05$ ). Similarly, other two modalities of CBCTthorax and CBCTpelvis had $10 \%$ less and below of CBCThead. The IGRT CBCT image reconstruction filters differed from their imaging protocols. This significant difference in the CBCT images might be due to the image reconstruction (Zhao et al., 2014; Rizz et al., 2005).

The results on the extracted four radiomics feature categories from the image sets of the phantom were

Table 2. Feature Extraction Algorithms Used in the Study with Estimated Feature Names

\begin{tabular}{|c|c|c|c|}
\hline Feature type & Feature Category & Estimated Feature Names & Comment \\
\hline $\begin{array}{l}\text { First order } \\
\text { Intensity }\end{array}$ & Intensity & $\begin{array}{l}\text { Energy, Entropy, Max, Mean, Median, Min, standard deviation, } \\
\text { Uniformity, Kurtosis, Skewness and Variance }\end{array}$ & \\
\hline \multirow[t]{3}{*}{ Texture } & $\begin{array}{l}\text { Gray level co-occurrence } \\
\text { matrix } 25 \\
(\text { GLCM) }\end{array}$ & $\begin{array}{l}\text { Autocorrelation, Cluster Prominence } \\
\text { Cluster Shade, Cluster Tendency, Contrast } \\
\text { Correlation, Difference Entropy, } \\
\text { Dissimilarity, Energy, Entropy, Homogeneity, } \\
\text { Homogeneity2, Information Measure Correlation1, } \\
\text { Information Measure Correlation } 2 \text {, Inverse Difference } \\
\text { Moment Normalised, Inverse Difference Normalised, Inverse } \\
\text { Variance, Max Probability, Sum Average, Sum Entropy Sum } \\
\text { Variance and Variance }\end{array}$ & $\begin{array}{l}25:=\text { GLCM is computed } \\
\text { from all } 2 \mathrm{D} \text { image slices }\end{array}$ \\
\hline & $\begin{array}{l}\text { Gray level run length } \\
\text { matrix (GLRLM) }\end{array}$ & $\begin{array}{l}\text { Gray Level Non-uniformity, High Gray Level Run Emphasis, } \\
\text { Long Runs Emphasis, Long Run High Gray Level Emphasis, } \\
\text { Long Run Low Gray Level Emphasis, Low Gray Level Run } \\
\text { Emphasis, Run Length Non-uniformity, Run Percentage, Short } \\
\text { Runs Emphasis, Short Run High Gray Level Emphasis, Short } \\
\text { Run Low Gray Level Emphasis. }\end{array}$ & \\
\hline & $\begin{array}{l}\text { Neighborhood intensity } \\
\text { difference matrix } 25 \\
\text { (NID) }\end{array}$ & $\begin{array}{l}\text { Busyness, Coarseness, Complexity, Contrast and Texture } \\
\text { Strength }\end{array}$ & $\begin{array}{l}25:=\text { Neighborhood } \\
\text { intensity different (NID) } \\
\text { is computed from all 2D } \\
\text { image slices }\end{array}$ \\
\hline
\end{tabular}


DOI:10.31557/APJCP.2021.22.5.1383

Analysing the Possibility of Utilizing CBCT Radiomics as an Independent Modality

Table 3. ICC Values Comparsion between CT and CBCT Radiomics Images

\begin{tabular}{|c|c|c|c|c|c|}
\hline Feature type & $\begin{array}{l}\text { Phantom image ROI } \\
\text { segment Density materials }\end{array}$ & $\begin{array}{c}\text { Average measures Intra class } \\
\text { Correlation } \\
\text { CT with CBCT radiomic features }\end{array}$ & $\begin{array}{l}\text { Significances } \\
\text { p-value }\end{array}$ & $\begin{array}{l}\text { 95\% Confidence } \\
\text { Interval } \\
\text { Lower Bound } \\
\end{array}$ & $\begin{array}{l}\text { Upper } \\
\text { Bound }\end{array}$ \\
\hline \multirow[t]{7}{*}{ Intensity } & Teflon & 0.902 & 0 & 0.787 & 0.963 \\
\hline & Delrin & 0.908 & 0 & 0.800 & 0.965 \\
\hline & acrylic & 0.895 & 0 & 0.773 & 0.961 \\
\hline & Polystyrene, & 0.896 & 0 & 0.774 & 0.961 \\
\hline & LDPE & 0.922 & 0 & 0.83 & 0.970 \\
\hline & PMP & 0.914 & 0 & 0.812 & 0.967 \\
\hline & Air & 0.91 & 0 & 0.805 & 0.966 \\
\hline \multirow[t]{7}{*}{ Texture GLCM } & Teflon & 0.952 & 0 & 0.909 & 0.978 \\
\hline & Delrin & 0.977 & 0 & 0.956 & 0.989 \\
\hline & acrylic & 0.999 & 0 & 0.997 & 0.999 \\
\hline & Polystyrene, & 0.995 & 0 & 0.990 & 0.998 \\
\hline & LDPE & 0.99 & 0 & 0.980 & 0.995 \\
\hline & PMP & 0.943 & 0 & 0.892 & 0.974 \\
\hline & Air & 0.958 & 0 & 0.921 & 0.981 \\
\hline \multirow{7}{*}{$\begin{array}{l}\text { Texture GL- } \\
\text { RLM }\end{array}$} & Teflon & 0.994 & 0 & 0.986 & 0.998 \\
\hline & Delrin & 0.995 & 0 & 0.989 & 0.999 \\
\hline & acrylic & 0.976 & 0 & 0.941 & 0.993 \\
\hline & Polystyrene, & 0.988 & 0 & 0.971 & 0.996 \\
\hline & LDPE & 0.996 & 0 & 0.991 & 0.999 \\
\hline & PMP & 0.997 & 0 & 0.992 & 0.999 \\
\hline & Air & 0.845 & 0 & 0.619 & 0.953 \\
\hline \multirow[t]{7}{*}{ Texture NID } & Teflon & 0.874 & 0.002 & 0.519 & 0.985 \\
\hline & Delrin & 0.827 & 0.008 & 0.343 & 0.980 \\
\hline & Acrylic & $0.418^{*}$ & 0.209 & -1.230 & 0.933 \\
\hline & Polystyrene, & 0.699 & 0.047 & -0.144 & 0.965 \\
\hline & LDPE & 0.824 & 0.008 & 0.332 & 0.980 \\
\hline & PMP & 0.84 & 0.006 & 0.391 & 0.982 \\
\hline & Air & $0.607 *$ & 0.093 & -0.491 & 0.954 \\
\hline
\end{tabular}

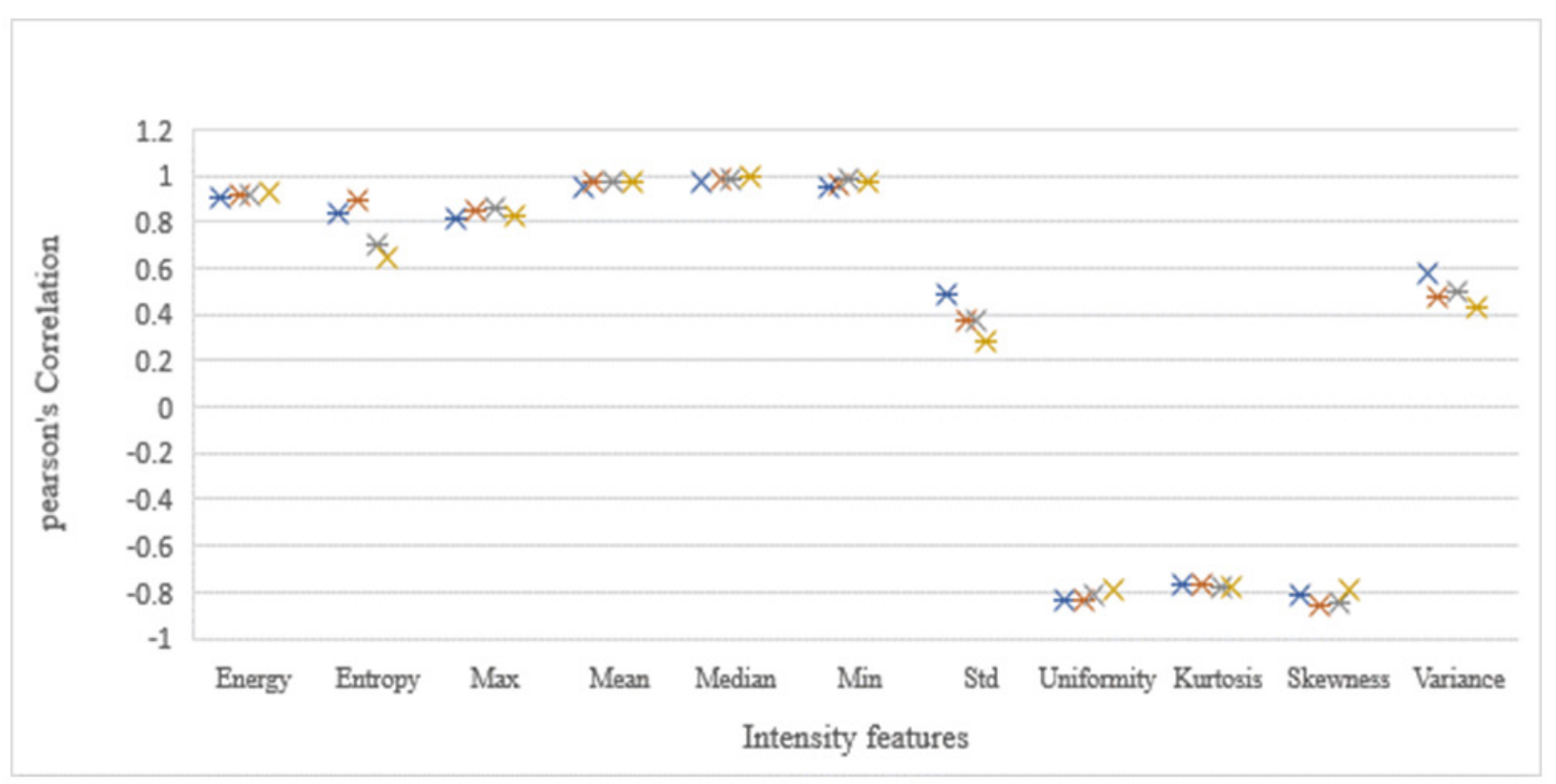

Figure 4a. Intensity Feature Relationship was Compared with Density Pearson's Correlation Coefficient in the Four Images and the Correlation Significances were Above $r=0.75$ and below $r=-0.75$. 
Table 4a. Radiomics Feature Variability with Respect to ROI Segment Density in Intensity Feature

\begin{tabular}{|c|c|c|c|c|c|c|c|c|}
\hline \multirow{2}{*}{$\begin{array}{l}\text { Intensity } \\
\text { features }\end{array}$} & \multicolumn{2}{|c|}{$\mathrm{CT}$} & \multicolumn{2}{|c|}{$\mathrm{CBCT}_{\text {head }}$} & \multicolumn{2}{|c|}{$\mathrm{CBCT}_{\text {thorax }}$} & \multicolumn{2}{|c|}{$\mathrm{CBCT}_{\text {pelvis }}$} \\
\hline & Pearson (r) & Sig p-value & Pearson (r) & Sig p-value & Pearson (r) & Sig p-value & Pearson (r) & Sig p-value \\
\hline${ }^{\mathrm{a} E n e r g y *}$ & 0.900 & 0.006 & 0.916 & 0.004 & 0.913 & 0.004 & 0.925 & 0.003 \\
\hline Entropy* & 0.837 & 0.019 & 0.897 & 0.006 & 0.699 & 0.081 & 0.647 & 0.116 \\
\hline${ }^{\mathrm{a}} \mathrm{Max}^{*}$ & 0.810 & 0.027 & 0.854 & 0.014 & 0.858 & 0.014 & 0.827 & 0.022 \\
\hline a'Mean* & 0.952 & 0.001 & 0.973 & 0.000 & 0.976 & 0.000 & 0.977 & 0.000 \\
\hline aMedian* & 0.969 & 0.000 & 0.98 & 0.000 & 0.986 & 0.000 & 0.990 & 0.000 \\
\hline${ }^{\mathrm{a}} \mathrm{Min} *$ & 0.946 & 0.001 & 0.965 & 0.000 & 0.985 & 0.000 & 0.970 & 0.000 \\
\hline Std & 0.488 & 0.267 & 0.370 & 0.413 & 0.370 & 0.414 & 0.283 & 0.539 \\
\hline anniformity* & -0.838 & 0.019 & -0.841 & 0.018 & -0.811 & 0.027 & -0.795 & 0.033 \\
\hline${ }^{\text {aKurtosis* }}$ & -0.764 & 0.046 & -0.766 & 0.045 & -0.774 & 0.041 & -0.774 & 0.041 \\
\hline aSkewness* & -0.813 & 0.026 & -0.854 & 0.015 & -0.845 & 0.017 & -0.794 & 0.033 \\
\hline Variance & 0.582 & 0.170 & 0.475 & 0.282 & 0.498 & 0.255 & 0.428 & 0.338 \\
\hline
\end{tabular}

a, indicates that the feature from the four image modalities had a strong correlation with significant $\mathrm{p}$-value $<0.05$; *, indicates that the feature from the four image modalities had a strong correlation with $r>0.5$

as follows. Out of the 11 intensity features, 8 showed significant correlation with $\mathrm{CT}$. The skewness and kurtosis of the intensity features category are used as a prognostic tools in identifying tumor genetic mutation of NSCLC (Weiss et al., 2014), while another study used skewness to predict overall survival (Ahn et al., 2015). Image features extracted from CBCT also act as an early potential biomarker for treatment response assessments (Bertelsen et al., 2011; Bernchou et al., 2015). Similarly, 5 of the 11 selected texture features of GLRLM had significant correlation with CT. The GLRLM feature expresses its usefulness in distinguishing benign lymph nodes from malignant ones (Bayanati et al., 2015). On the other hand, among the 22 GLCM features, 9 had strong correlation with $\mathrm{CT}$, which is in line with findings of a previous study calculated the CT images using an anthropomorphic which phantom (Mahmood et al., 2017). However, in a clinical study, Coroller et al., considered this feature to the best radiomics feature in predicting distant metastasis in NSCLC patients (Coroller et al., 2015).

Table 4b. Radiomics Texture Feature Variability with Respect to ROI Segment Density in GLCM Feature

\begin{tabular}{|c|c|c|c|c|c|c|c|c|}
\hline \multirow{2}{*}{$\begin{array}{l}\text { Gray Level Co } \\
\text { occurrence Matrix feature }\end{array}$} & \multicolumn{2}{|c|}{$\mathrm{CT}$} & \multicolumn{2}{|c|}{$\mathrm{CBCT}_{\text {head }}$} & \multicolumn{2}{|c|}{$\mathrm{CBCT}_{\text {thorax }}$} & \multicolumn{2}{|c|}{$\mathrm{CBCT}_{\text {pelvis }}$} \\
\hline & Pearson (r) & Sig p-value & Pearson (r) & Sig p-value & Pearson (r) & Sig p-value & Pearson (r) & Sig p-value \\
\hline aAuto Correlation* & 0.903 & 0.005 & 0.925 & 0.003 & 0.92 & 0.003 & 0.926 & 0.003 \\
\hline Cluster Prominence* & 0.610 & 0.146 & 0.601 & 0.154 & 0.609 & 0.146 & 0.553 & 0.198 \\
\hline aCluster Shade* & -0.859 & 0.013 & -0.844 & 0.017 & -0.832 & 0.020 & -0.903 & 0.005 \\
\hline Cluster Tendency & 0.546 & 0.205 & 0.516 & 0.236 & 0.526 & 0.226 & 0.427 & 0.339 \\
\hline Contrast & 0.642 & 0.120 & 0.410 & 0.36 & 0.438 & 0.326 & 0.45 & 0.311 \\
\hline Correlation & -0.231 & 0.618 & 0.565 & 0.186 & 0.482 & 0.273 & 0.088 & 0.851 \\
\hline Difference Entropy & 0.729 & 0.063 & 0.639 & 0.122 & 0.378 & 0.403 & 0.299 & 0.514 \\
\hline Dissimilarity & 0.701 & 0.08 & 0.417 & 0.352 & 0.441 & 0.321 & 0.389 & 0.389 \\
\hline aEnergy* & -0.806 & 0.028 & -0.815 & 0.026 & -0.797 & 0.032 & -0.768 & 0.044 \\
\hline Entropy* & 0.784 & 0.037 & 0.883 & 0.008 & 0.638 & 0.123 & 0.571 & 0.180 \\
\hline Homogeneity & -0.782 & 0.038 & -0.877 & 0.01 & -0.604 & 0.151 & -0.449 & 0.313 \\
\hline Homogeneity2 & -0.758 & 0.049 & -0.882 & 0.009 & -0.582 & 0.171 & -0.411 & 0.359 \\
\hline InformationMeasureCorr1 & 0.169 & 0.718 & -0.548 & 0.203 & -0.238 & 0.607 & -0.272 & 0.555 \\
\hline InformationMeasureCorr2 & 0.153 & 0.744 & 0.581 & 0.171 & 0.299 & 0.515 & 0.256 & 0.580 \\
\hline Inverse Diff Moment Norm & -0.642 & 0.12 & -0.403 & 0.37 & -0.434 & 0.330 & -0.445 & 0.317 \\
\hline Inverse Diff Norm & -0.706 & 0.076 & -0.42 & 0.348 & -0.441 & 0.322 & -0.378 & 0.404 \\
\hline Inverse Variance & 0.164 & 0.725 & 0.292 & 0.526 & 0.042 & 0.929 & 0.154 & 0.742 \\
\hline${ }^{a}$ Max Probability* & -0.803 & 0.03 & -0.808 & 0.028 & -0.802 & 0.03 & -0.783 & 0.037 \\
\hline 'Sum Average* & 0.951 & 0.001 & 0.974 & 0.000 & 0.977 & 0.000 & 0.977 & 0.000 \\
\hline Sum Entropy* & 0.806 & 0.029 & 0.814 & 0.026 & 0.631 & 0.129 & 0.557 & 0.194 \\
\hline 'Sum Variance* & 0.902 & 0.006 & 0.922 & 0.003 & 0.919 & 0.003 & 0.926 & 0.003 \\
\hline Variance & 0.546 & 0.205 & 0.516 & 0.236 & 0.526 & 0.226 & 0.427 & 0.339 \\
\hline
\end{tabular}

a , indicates that the feature from the four image modalities had a strong correlation with significant $\mathrm{p}$-value $<0.05$; *, indicates that the feature from the four image modalities had a strong correlation with $r>0.5$ 
DOI:10.31557/APJCP.2021.22.5.1383

Analysing the Possibility of Utilizing CBCT Radiomics as an Independent Modality

Table 4c. Radiomics Texture Feature Variability with Respect to ROI Segment Density in GLRLM Feature

\begin{tabular}{|c|c|c|c|c|c|c|c|c|}
\hline \multirow{2}{*}{$\begin{array}{l}\text { Gray Level Run Length Matrix } \\
\text { Features }\end{array}$} & \multicolumn{2}{|c|}{$\mathrm{CT}$} & \multicolumn{2}{|c|}{$\mathrm{CBCT}_{\text {head }}$} & \multicolumn{2}{|c|}{$\mathrm{CBCT}_{\text {thorax }}$} & \multicolumn{2}{|c|}{$\mathrm{CBCT}_{\text {pelvis }}$} \\
\hline & Pearson (r) & Sig p-value & Pearson (r) & Sig p-value & Pearson (r) & Sig p-value & Pearson (r) & Sig p-value \\
\hline Gray Level Non uniformity & -0.044 & 0.925 & -0.76 & 0.048 & 0.072 & 0.879 & -0.260 & 0.573 \\
\hline aHigh Gray Level Run Emphasis* & 0.883 & 0.008 & 0.906 & 0.005 & 0.906 & 0.005 & 0.911 & 0.004 \\
\hline Long Run Emphasis* & -0.797 & 0.032 & -0.809 & 0.028 & -0.802 & 0.030 & -0.737 & 0.059 \\
\hline $\begin{array}{l}\text { Long Run High Gray Level } \\
\text { Emphasis }\end{array}$ & -0.316 & 0.490 & 0.725 & 0.065 & 0.658 & 0.108 & 0.772 & 0.042 \\
\hline $\begin{array}{l}\text { aLong Run Low Gray Level } \\
\text { Emphasis* }\end{array}$ & -0.820 & 0.024 & -0.827 & 0.022 & -0.821 & 0.024 & -0.832 & 0.020 \\
\hline 'Low Gray Level Run Emphasis* & -0.989 & 0.000 & -0.955 & 0.001 & -0.944 & 0.001 & -0.948 & 0.001 \\
\hline Run Length Non uniformity & 0.721 & 0.068 & 0.862 & 0.013 & 0.697 & 0.082 & 0.483 & 0.272 \\
\hline Run Percentage* & 0.767 & 0.044 & 0.836 & 0.019 & 0.765 & 0.045 & 0.608 & 0.147 \\
\hline Short Run Emphasis & 0.749 & 0.053 & 0.857 & 0.014 & 0.621 & 0.136 & 0.418 & 0.351 \\
\hline $\begin{array}{l}\text { aShort Run High Gray Level } \\
\text { Emphasis* }\end{array}$ & 0.894 & 0.007 & 0.906 & 0.005 & 0.893 & 0.007 & 0.897 & 0.006 \\
\hline $\begin{array}{l}\text { aShort Run Low Gray Level } \\
\text { Emphasis* }\end{array}$ & -0.816 & 0.025 & -0.980 & 0.000 & -0.966 & 0.000 & -0.965 & 0.000 \\
\hline
\end{tabular}

a, indicates that the feature from the four image modalities had a strong correlation with significant $p$-value $<0.05$; *, indicates that the feature from the four image modalities had a strong correlation with $r>0.5$

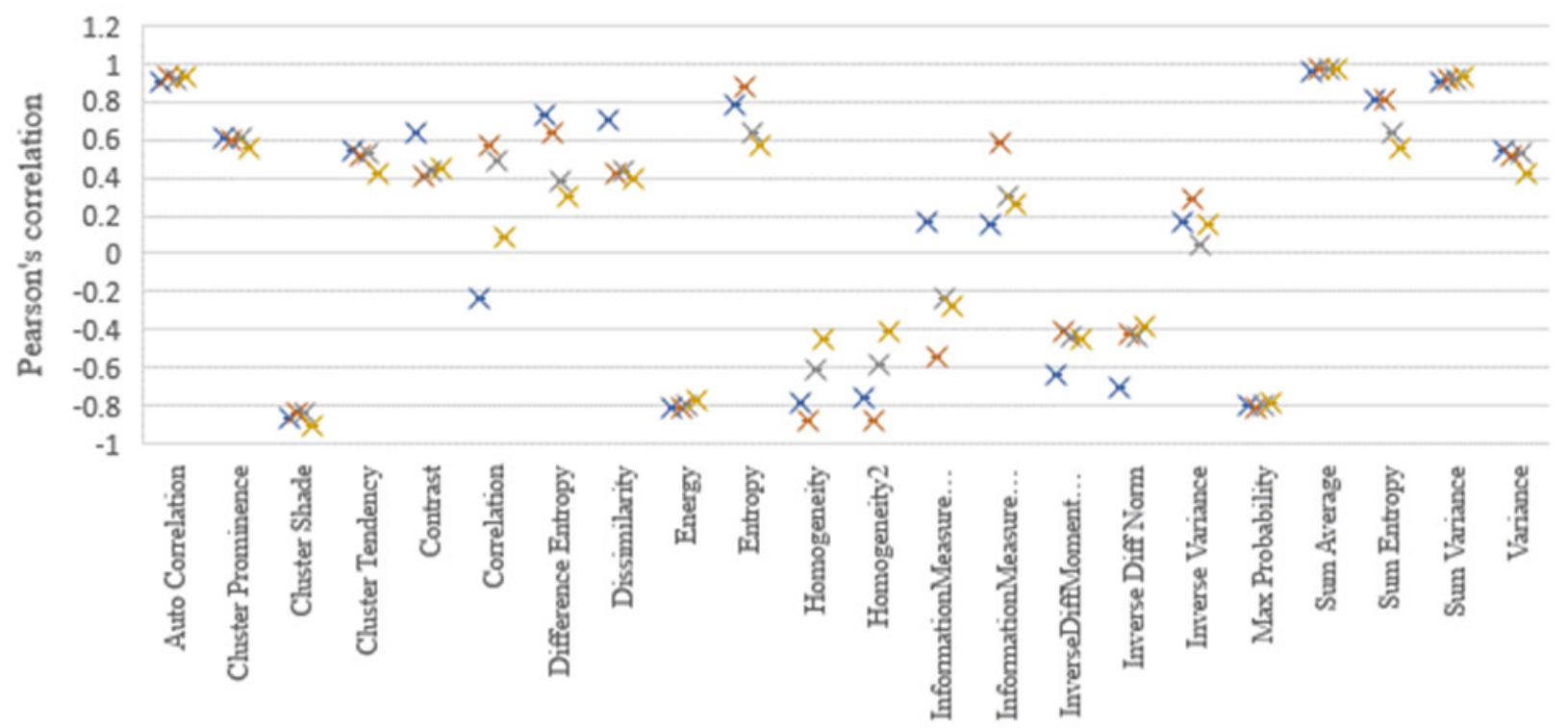

Gray Level Co-occurrence Matrix

Figure 4b. GLCM Feature Relationship was Compared with Density Pearson's Correlation Coefficient in the Four Images and the Correlation Significances were Above $r=0.75$ and below $r=-0.75$

Finally, out of 5 NID features, only one feature showed strong correlation with $\mathrm{CT}$, which is in accordance with findings of Mahmood et al.'s study. Thus, we found that the intensity and the GLRLM feature category of the CT were reproducible by the radiomics features of $\mathrm{CBCT}$. The other two feature categories of the CT, namely GLCM and NID, which were considered not reproducible across scanners even under idealized circumstances remained

Table 4d. Radiomics Texture Feature Variability with Respect to ROI Segment Density in NID Feature

\begin{tabular}{lcccccccc}
\hline \multirow{2}{*}{$\begin{array}{l}\text { Neighbor Intensity } \\
\text { Difference Feature }\end{array}$} & \multicolumn{2}{c}{$\mathrm{CT}$} & \multicolumn{2}{c}{$\mathrm{CBCT}_{\text {head }}$} & \multicolumn{2}{c}{$\mathrm{CBCT}_{\text {thorax }}$} & \multicolumn{2}{c}{$\mathrm{CBCT}_{\text {pelvis }}$} \\
& Pearson (r) & Sig p-value & Pearson (r) & Sig p-value & Pearson (r) & Sig p-value & Pearson (r) & Sig p-value \\
\hline Busyness & 0.07 & 0.881 & -0.453 & 0.307 & 0.031 & 0.947 & -0.237 & 0.609 \\
Coarseness & -0.787 & 0.036 & -0.244 & 0.599 & -0.603 & 0.152 & -0.129 & 0.783 \\
Complexity* & 0.68 & 0.093 & 0.549 & 0.202 & 0.658 & 0.108 & 0.54 & 0.211 \\
Contrast & 0.743 & 0.056 & -0.048 & 0.918 & 0.669 & 0.1 & 0.468 & 0.289 \\
Texture Strength & -0.103 & 0.827 & 0.428 & 0.338 & 0.121 & 0.796 & 0.06 & 0.899 \\
\hline
\end{tabular}

${ }^{a}$, indicates that the feature from the four image modalities had a strong correlation with significant $p$-value $<0.05 ; *^{*}$, indicates that the feature from the four image modalities had a strong coefficient with $r>0.5$ 


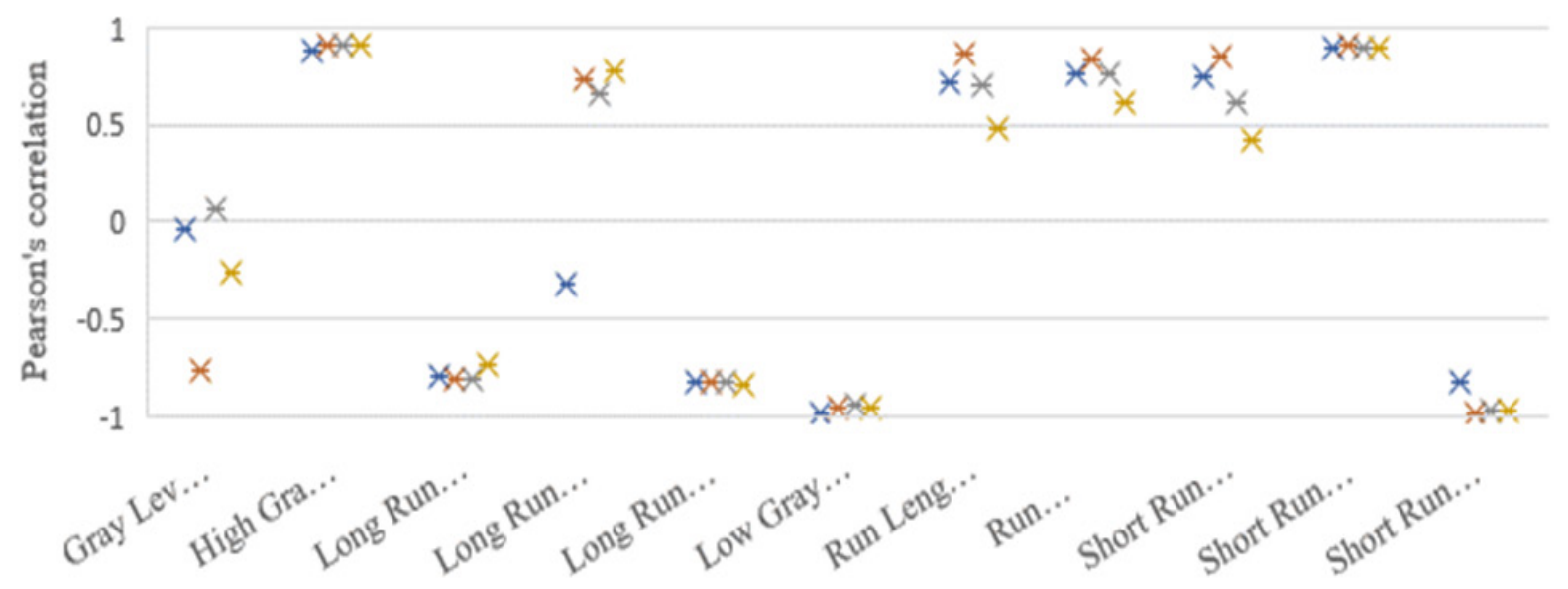

Gray Level Run Length Matrix

Figure 4c. GLRLM Feature Relationship was Compared with Density Pearson's Correlation Coefficient in the Four Images and the Correlation Significances were Above $r=0.75$ and Below $r=-0.75$

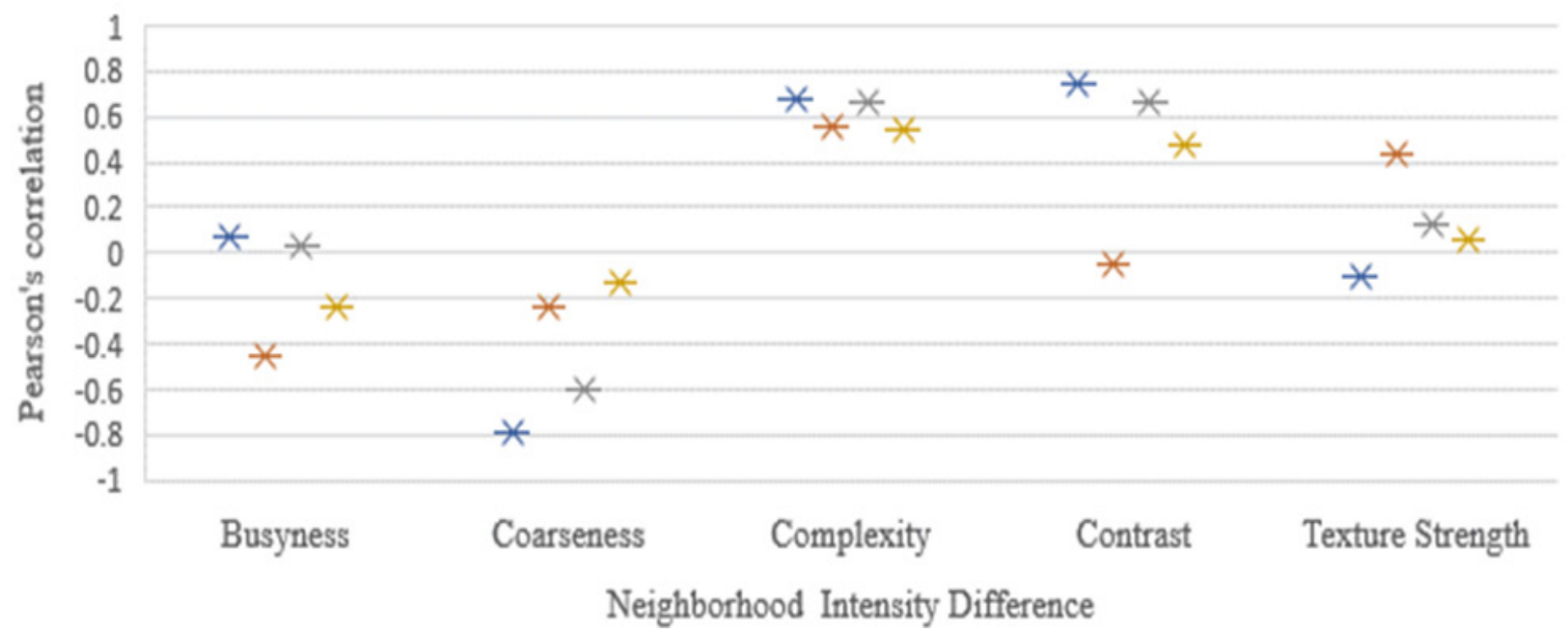

Figure 4d. NID Feature Relationship was Compared with Density Pearson's Correlation Coefficient in the Four Images and the Correlation Significances were Above $r=0.75$ and Below $r=-0.75$.

the same for the CBCT radiomics features.

ICC was calculated to measure the similarities of each feature category between the seven density materials of the $C T$ and $C B C T$

This analysis demonstrated reproducible feature category from different density image segments among the four scans. ICC of the images in the dissimilar mediums of four selected feature categories was compared in box plot wispier. In this comparison, the interquartile range of NID differed from the other three feature categories. The density materials of NID, namely Acrylic and Air, ROIs $\mathrm{ICC}=0.418, \mathrm{P}=0.209, \mathrm{ICC}=0.607, \mathrm{P}=0.09$ demonstrating non-significant $\mathrm{p}$ - value. This is because of less uniformity in ROIs physical density (Fave et al., 2015). NID texture features are calculated from the spatial distribution of voxel intensities or CT numbers. Moreover, CBCT is an ancillary modality and considered for image guidance, its pixel units values are likely less accurate than $\mathrm{CT}$ pixel units and $\mathrm{CBCT}$ pixel mapping differs from $\mathrm{CT}$ images. In addtion, CBCT image formation method is different from CT modality.

In a nutshell, $39 \%$ of the 49 selected computed features had strong and significant correlation with CT. While the remaining $61 \%$ features, had low and medium correlation. These findings further substantiated the probabilities of interrelation amongst the imaging modalities of discrete density material textures, thereby incrementing the reliability of our study. According to the ICC results, the four feature categories, namely intensity, GLCM, and GLRLM, showed significance/significant $\mathrm{p}$ value. This range of reproducible features can be helpful to provide unique information from anatomical tissues. However, the clinical studies showed different outcomes as compared to phantom study. Therefore, further studies correlating clinical with phantom studies would probably clarify the present limitations and possibly aid in better understanding of the applications of CBCT radiomics. In conclusion, as $\mathrm{CBCT}$ images are taken regularly, we had considered it for our study. According to findings of this phantom study, CBCT radiomics could probably be considered as an independent modality. 


\section{Author Contribution Statement}

The authors confirm contribution to the paper as follows: study conception and design: Dharmenrdan palani $1^{\text {st }}$ Author; data collection: Dharmendran palani $1^{\text {st }}$ Author, Kesavan Govindaraj $3^{\text {rd }}$ Author ; analysis and interpretation of results: Dharmenran palani 1st Author, Senthilkumar Shanmugam $2^{\text {nd }}$ Author, Kesavan Govindaraj $3^{\text {rd }}$ Author; draft manuscript preparation: Dharmendran palani 1st Author, Senthilkumar Shanmugam $2^{\text {nd }}$ Author. All authors reviewed the results and approved the final version of the manuscript.

\section{Acknowledgments}

None.

\section{Conflict of interest}

No conflict of interest

\section{References}

Aerts H, Velazquez E, Leijenaar R, et al (2014). Decoding tumour phenotype by noninvasive imaging using a quantitative radiomics approach. Nat Commun, 5, 4006.

Ahn SY, Park CM, Park SJ, et al (2015). Prognostic value of computed tomography texture features in non-small cell lung cancers treated with definitive concomitant chemoradiotherapy. Invest Radiol, 50, 719-25.

Bayanati H, E Thornhill R, Souza CA, et al (2015). Quantitative $\mathrm{CT}$ texture and shape analysis: can it differentiate benign and malignant mediastinal lymph nodes in patients with primary lung cancer?. Eur Radiol, 25, 480-7.

Bernchou U, Hansen O, Schytte T, et al (2015). Prediction of lung density changes after radiotherapy by cone beam computed tomography response markers and pre-treatment factors for non-small cell lung cancer patients. Radiother Oncol, 117, 17-22.

Bertelsen A, Schytte T, Bentzen SM, et al (2011). Radiation dose response of normal lung assessed by Cone Beam CT - a potential tool for biologically adaptive radiation therapy. Radiother Oncol, 100, 351-5.

Brink C, Bernchou U, Bertelsen A, et al (2014). Locoregional control of non-small cell lung cancer in relation to automated early assessment of tumor regression on cone beam computed tomography. Int J Radiat Oncol Biol Phys, 89, 916-23.

Bundschuh RA, Dinges J, Neumann L, et al (2014). Textural parameters of tumor heterogeneity in ${ }^{1} \mathrm{~F}-\mathrm{FDG} \mathrm{PET} / \mathrm{CT}$ for therapy response assessment and prognosis in patients with locally advanced rectal cancer. $J$ Nucl Med, 55, 891-7.

Coroller TP, Grossmann P, Hou Y, et al (2015). CT-based radiomic signature predicts distant metastasis in lung adenocarcinoma. Radiother Oncol, 114, 345-50.

De Cecco CN, Ganeshan B, Ciolina M, et al (2015). Texture analysis as imaging biomarker of tumoral response to neoadjuvant chemoradiotherapy in rectal cancer patients studied with 3-T magnetic resonance. Invest Radiol, 50, 239-45.

Fave X, Mackin D, Yang J, et al (2015). Can radiomics features be reproducibly measured from CBCT images for patients with non-small cell lung cancer?. Med Phys, 42, 6784-97.

Fried DV, Tucker SL, Zhou S, et al (2014). Prognostic value and reproducibility of pretreatment CT texture features in stage III non-small cell lung cancer. Int J Radiat Oncol Biol
Phys, 90, 834-42.

Gillies RJ, Kinahan PE, Hricak H (2016). Radiomics: Images are more than pictures, they are data. Radiology, 278, 563-77.

Haralick RM , shanmugam K , Dinstein I (1973). Textural features for image classification. IEEE Trans Syst Man Cybernet, 3, 610-21.

Lambin P, Leijenaar RTH, Deist TM, et al (2017). Radiomics: the bridge between medical imaging and personalized medicine. Nat Rev Clin Oncol, 14, 749-62.

Mahmood U, Apte AP, Deasy JO, Schmidtlein CR, ShuklaDave A (2017). Investigating the robustness neighborhood gray tone difference matrix and gray level co-occurrence matrix radiomic features on clinical computed tomography systems using anthropomorphic phantoms: Evidence from a multivendor study. J Comput Assisted Tomography, 41, 995-1001.

Nardone V, Tini P, Biondi M, et al (2016). Prognostic value of MR imaging texture analysis in Brain non-small cell lung cancer Oligo-Metastases undergoing stereotactic irradiation. Cureus, 8, e584. Published 2016 Apr 25.

Nie K, Shi L, Chen Q, et al (2016). Rectal cancer: Assessment of neoadjuvant chemoradiation outcome based on radiomics of multiparametric MRI. Clin Cancer Res, 22, 5256-64.

Pyka T, Bundschuh RA, Andratschke N, et al (2015). Textural features in pre-treatment [F18]-FDG-PET/CT are correlated with risk of local recurrence and disease-specific survival in early stage NSCLC patients receiving primary stereotactic radiation therapy. Radiat Oncol, 10, 100.

Rizzo SM, Kalra MK, Schmidt B, et al (2005). CT images of abdomen and pelvis: effect of nonlinear three-dimensional optimized reconstruction algorithm on image quality and lesion characteristics. Radiology, 237, 309-15.

Tian F, Hayano K, Kambadakone AR, Sahani DV (2015). Response assessment to neoadjuvant therapy in soft tissue sarcomas: using CT texture analysis in comparison to tumor size, density, and perfusion. Abdom Imaging, 40, 1705-12.

Weiss GJ, Ganeshan B, Miles KA, et al (2014). Noninvasive image texture analysis differentiates K-ras mutation from pan-wildtype NSCLC and is prognostic. PLoS One, 9, e100244.

Yip C, Landau D, Kozarski R, et al (2014). Primary esophageal cancer: heterogeneity as potential prognostic biomarker in patients treated with definitive chemotherapy and radiation therapy. Radiology, 270, 141-8.

Zhao B, Tan Y, Tsai WY, Schwartz LH, Lu L (2014). Exploring variability in CT characterization of tumors: A Preliminary Phantom Study. Transl Oncol, 1, 88-93.

\section{(ब) $(\mathbb{Q} \otimes$}

This work is licensed under a Creative Commons AttributionNon Commercial 4.0 International License. 